\title{
Establishing a magnetic resonance safety program
}

\author{
Susan T. Sotardi ${ }^{1}$ - Andrew J. Degnan ${ }^{1}$ • Chang Amber Liu ${ }^{2}$ • Patricia L. Mecca ${ }^{1}$ - Suraj D. Serai ${ }^{1}$ - R. Daniel Smock ${ }^{3}$. \\ Teresa Victoria ${ }^{1} \cdot$ Ammie M. White ${ }^{1}$
}

Received: 1 July 2020 / Revised: 3 September 2020 / Accepted: 9 November 2020/ Published online: 15 April 2021

(C) Springer-Verlag GmbH Germany, part of Springer Nature 2021

\begin{abstract}
Establishing a magnetic resonance (MR) safety program is crucial to ensuring the safe MR imaging of pediatric patients. The organizational structure includes a core safety council and broader safety committee comprising all key stakeholders. These groups work in synchrony to establish a strong culture of safety; create and maintain policies and procedures; implement device regulations for entry into the MR setting; construct MR safety zones; address intraoperative MR concerns; guarantee safe scanning parameters, including complying with specific absorption rate limitations; adhere to national regulatory body guidelines; and ensure appropriate communication among all parties in the MR environment. Perspectives on the duties of the safety council members provide important insight into the organization of program oversite. Ultimately, the collective dedication and vigilance of all MR staff are crucial to the success of a safety program.
\end{abstract}

Keywords Children · Conditional devices $\cdot$ Guidelines $\cdot$ Magnetic resonance imaging $\cdot$ Quality $\cdot$ Safety $\cdot$ Safety program

\section{Introduction}

Ensuring a safe environment during MRI is paramount for any imaging center. Therefore, the establishment and maintenance of a robust magnetic resonance (MR) safety program is vital to safeguard the well-being of patients and MR personnel. Creating such a safety program to meet the distinct needs of pediatric patients is multi-faceted and differs from doing so in adult programs, in several important respects. Children often cannot provide their own history, cannot give consent, and might not be able to articulate their concerns. Thus, pediatric providers must be adept in communicating with both children and their caregivers when performing risk screening and planning for an MRI. Family members or other clinical personnel often accompany children undergoing an MRI; as such, we must also consider the MR safety concerns related to everyone accompanying pediatric

Susan T. Sotardi

sotardis@email.chop.edu

1 Department of Radiology, Children's Hospital of Philadelphia, 3401 Civic Center Blvd., Philadelphia, PA 19104, USA

2 Department of Anesthesiology, Massachusetts General Hospital, Boston, MA, USA

3 Department of Radiology, Children's Mercy Hospital, Kansas City, MO, USA patients into the scanner room. Child life professionals provide an important service for young children undergoing relatively short MR exams. However, in contrast to adult populations, children more often require sedation or anesthesia services, resulting in concerns about the use of anesthesia in young children $[1,2]$. Furthermore, while commercially available MR conditional support equipment is available for adults, the equipment options are far more limited for the pediatric population, requiring an understanding of appropriate technical modifications and alternatives. Accounting for these complex challenges, the mandate of MR safety programs is to facilitate a strong culture of safety, establish and maintain policies and procedures that ensure safe practice, educate personnel about safety-related policies and procedures, provide consultations for difficult situations, and manage any safety incidents in real time.

Creating a team that functions to address these duties appropriately and expeditiously is crucial to the success of the program [3]. This requires involving representatives from all the key stakeholders regularly practicing in the MR environment including, but not limited to, radiologists, MR technologists, radiology nurses, anesthesiologists and sedation providers, cardiologists and child life specialists. However, MR imaging often requires participation by hospital staff who do not regularly work in the MR environment, might not appreciate the level of risk posed by the magnet, and might not be accustomed to standard MR operating procedures. Additionally, the entry of family members and non-MR personnel into the MR 
environment must be considered in a comprehensive and strategic manner. To ensure a safe environment, each of these parties must be sufficiently informed to act in the best interest of everyone's safety. All must feel comfortable asking questions and discussing potential concerns with team members at all levels, without fear of repercussions $[4,5]$.

\section{Program structure}

The Inter-Society Working Group on MR Safety has issued recommendations on the organizational structure for the management of MR safety [6]. At the authors' institutions, the MR safety programs are composed of an MR Safety Council (the Council) and an MR Safety Committee (the Committee). The Council consists of radiologists who might obtain MR medical director (MRMD) certification, senior MR technologists with expertise in MR safety who might obtain MR safety officer (MRSO) certification, and an MR safety expert (MRSE) who is an MR physicist. These positions have been established by the American Board of Magnetic Resonance Safety (ABMRS) toward developing clear safety roles and responsibilities. The Council provides oversight and leadership of the MR safety program. The members are available to assist with operations of the MR system at all times during which the MR facility is accessible to patients.

The Council serves to create and maintain departmental safety policies and to implement associated procedures. The Council members are available for consultation regarding any patient-specific questions that arise about devices or other potential safety issues. They are also on call for immediate emergency response to safety incidents. Furthermore, the Council performs a root-cause analysis in the event of any safety incident. It is crucial that all members involved in the performance of MRI feel comfortable escalating concerns or requesting assistance from the Council as part of a culture of safety [5].

The Committee comprises a larger group, including the Council members, with representatives from all the key stakeholder areas, some of which were outlined earlier, as well as research MR personnel and others that might differ by institution. There might be ad hoc participation by other key clinical areas, such as the critical care units, as needed. In light of the multitude of team members' viewpoints, the Committee is a necessary method for sharing and integrating the unique perspectives of all team members. The committee members serve as liaisons with their respective sections, advocate on behalf of patients and their colleagues and provide feedback concerning policy implementation and areas for improvement. To facilitate the effective performance of the safety-related tasks of an MR department, the Committee acts as a managing body that offers guidance and supervision to the department and institution regarding the safe conduct of MRI examinations.
The Committee meets regularly to review and ratify policies and procedures related to MR safe practice, to discuss procedural updates and practice changes (most frequently related to new device safety recommendations), to consider ongoing workflow optimization, to discuss progress toward ongoing improvement efforts, and to report any recent safety incidents or near-misses. The Committee also provides a forum for open discussion, during which members may raise concerns, collaborate to address areas of safety vulnerability, build consensus around challenging issues, and suggest topics for further examination.

Safety activities should also be maintained daily. Examples of daily activities include a morning safety huddle and ongoing technologist screening programs. The morning readiness huddle follows the S-MESA (safety, methods, equipment, supplies or associates) format advocated by others [7, 8]. All departmental staff should be encouraged to participate, including technologists, nurses, anesthesiologists, sedation teams, radiologists and child life specialists. The huddle addresses topics pertaining to daily clinical workflow activities, such as patient-specific concerns for the day, follow-up about prior incidents or issues, and discussion of new workflow solutions and procedures. Additionally, technologists are responsible for screening future scheduled patients for the presence of retained metal or implanted devices.

\section{Procedure creation and maintenance}

One of the primary functions of the MR safety program is the creation and management of policies and procedures related to MR safe practice. These policies and procedures should be publicly available on an institution-wide network for easy reference. These policies and procedures are reviewed at regular intervals to incorporate changes from regulatory bodies, departmental or hospital procedural changes, as well as recommendations from the larger Committee. The topics of these policies and procedures include a wide range of subject matter, such as patient and staff screening, implantable electronic devices and other scanning parameters, and emergency response plans. As discussed, these policies must integrate the needs of all team members. For example, managing codes in the MR environment poses a distinct hazard to both patient and staff safety and therefore must be managed differently from how it is in other clinical areas. Standard processes for code management within the MR environment should be developed in conjunction with anesthesiologists, the sedation team and radiology nursing colleagues, in agreement with American College of Radiology (ACR) recommendations for safe practice. The incorporation of each committee member's perspective strengthens these procedures to withstand the challenges of managing a safety incident, thereby mitigating the risk of harm to patients and personnel. 


\section{Devices}

One of the most important aspects of an MR safety program is managing device entry into the MR environment to decrease potential risks from the powerful static magnetic field, as well as time-varying gradient and radiofrequency magnetic fields. While some of these dangers are clearly documented for individual implanted devices, many medical devices lack clear labels with respect to MR safety profiles. Therefore, the entry of any device into the MR environment must have a standardized process for documentation, approval and use. To accurately assess the safety profile of a device, the screening process must begin well in advance of the patient's arrival in the MR department. At the authors' institutions, screening begins at the time of order placement. A template for MR screening forms is available through the ACR safety website. Clinicians confirm the presence of implants, or lack thereof, during order entry. Next, outpatient schedulers pre-screen from a standard list of potentially problematic devices, so that these can be investigated and cleared before scheduling any MRI. If no such device is present, the patient is scheduled for an exam, but also undergoes thorough MR safety screening after arriving in the radiology department on the day of imaging. In the event that an unanticipated implantable device is identified, an MR technologist will investigate the device and its safety profile before the MRI can proceed. If a device cannot be cleared by the MR technologist, the MR safety officer assumes responsibility and might consult with the MR medical director and MR safety expert for more advanced risk assessment and clearance.

\section{Zone implementation}

The ACR provides guidelines for distinguishing physical areas, or zones, in relation to their proximity to the MR magnet, each of which confers different levels of risk (Fig. 1). Four safety zones have been designated, with zone 1 being the least restrictive and zone 4 the most restrictive. Zone 1 constitutes general public areas outside the MR environment. Zone 2 offers a buffer before the MRI control room (zone 3) and MR scanner room (zone 4), including all possible routes to or from the MR scanning area. Moving between zone 2 and zone 3 requires badge-restricted access and security level permissions conferred by specialized training. Non-MR personnel may only enter zone 3 under direct supervision. Zone 3 is contiguous with the scanner room and requires equivalent security restrictions as zone 4 . Access to zones 3 and 4 requires all electronic and ferromagnetic materials be removed prior to entry. Moving from zone 3 into zone 4 involves passing through a shielded door with an alarmed ferromagnetic detection system and a physical barrier (e.g., plastic chain across the doorway) that provides a last reminder to check for ferromagnetic items. Zone implementation provides crucial structure to limit the activities permitted within each area. Most important, emergency situations within zone 4, the MR suite, must be approached by quickly removing the patient from zone 4 before any resuscitation is initiated.

\section{Intraoperative magnetic resonance considerations}

Intraoperative MRI refers to either an operating room (OR) with an MR scanner or the transport of a patient to a nearby MR suite during a pause in surgery. Because intraoperative MRI improves surgical outcomes, use of intraoperative imaging has increased in recent years [9]. However, intraoperative MRI comes with numerous challenges for patient safety. Intraoperative MRI performed within an operating room has important consequences for all OR equipment, which must be tested for MR safety and potentially retested to comply with maintenance standards at predetermined intervals [10]. Instruments approved for use within the 5-gauss line are typically non-ferromagnetic or low iron-content steel and specifically intended for use in an MR environment. Training of any personnel in an OR suite with an MR scanner must include safety certification [10]. Because installing and maintaining an MR scanner in the OR poses significant challenges and expense, with expected low use, many institutions opt to provide intraoperative imaging in a nearby MRI suite. However, transporting an anesthetized child from the operating room to a separate MRI area has additional logistical challenges for the clinical teams [11]. Anesthesiologists, nurses, surgeons and other clinical team members must ensure safe transport to and from the operating room. The screening process for intraoperative patients requires meticulous accounting of surgical and anesthesiology equipment prior to entry into zones 3 and 4. Checklists have been shown to be helpful in the intraoperative MRI environment [12]. But while checklists provide another layer of safety assurance, particularly in uncommon situations, there is no replacement for clear and methodical reasoning on the part of all team members. In addition, radiologist workflows must accommodate rapid real-time assessment during imaging to determine whether return to surgery is necessary.

\section{Specific absorption rate limitations}

Policies must be in place to limit energy deposition into the patient during MRI. There are two commonly used metrics that characterize the amount of radiofrequency energy that is absorbed by the human body: specific absorption rate (SAR) and specific energy dose (SED). While the SAR metric is 
Fig. 1 Diagram shows the American College of Radiology's four MRI safety zones in relation to their proximity to the MR magnet; each zone confers a different level of risk

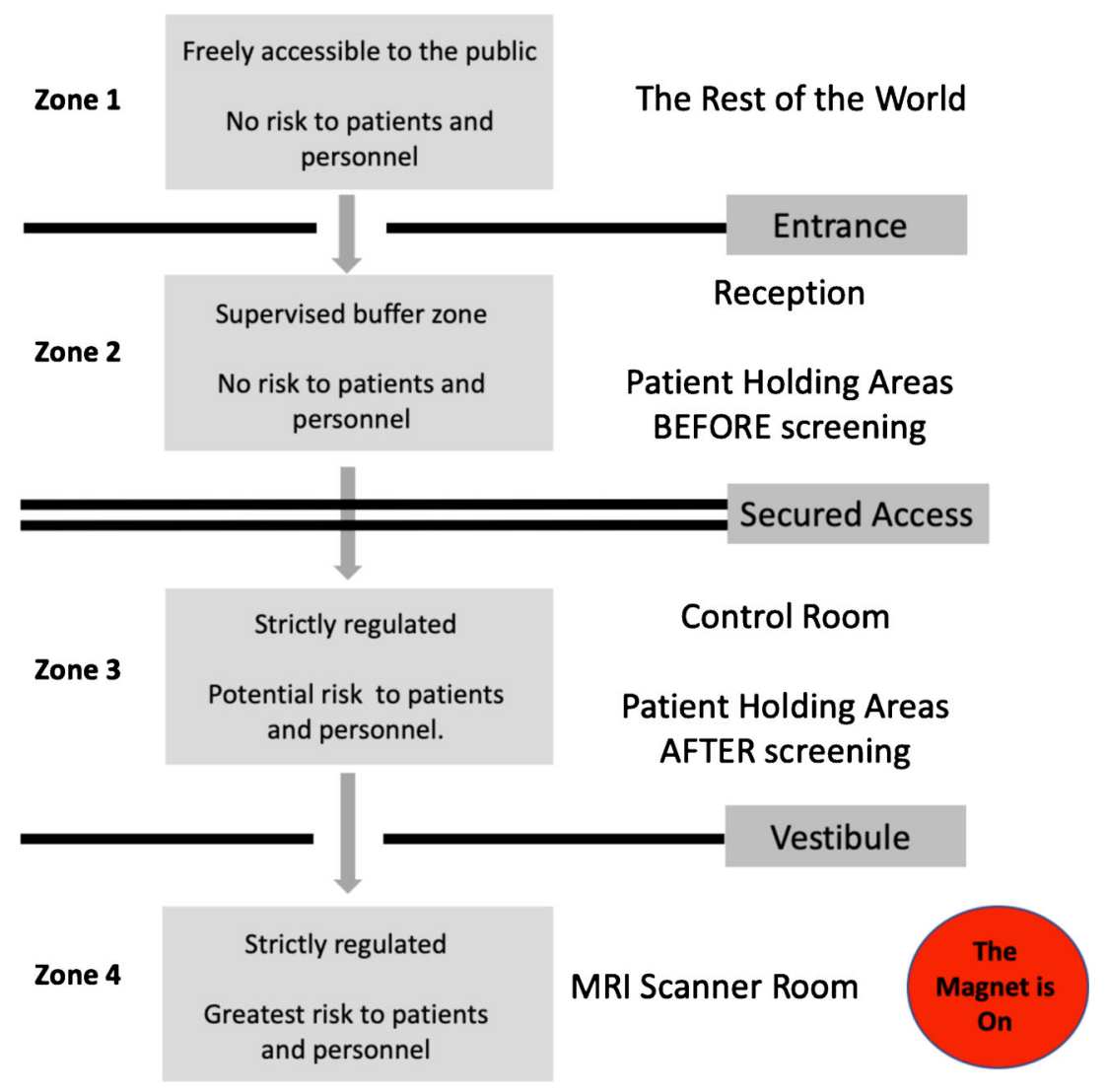

more commonly monitored during the scan, several manufacturers have recently implemented SED limits on their MR scanners in an effort to prevent excessive temperatures associated with long-duration or high-SAR pulse sequences. The United States Food and Drug Administration (FDA) sets strict limits on individual exposure to a certain power deposition, with an upward SAR limit of $4 \mathrm{~W} / \mathrm{kg}$ on a first-level controlled MRI mode for the body. The International Electrotechnical Commission has set an SED limit of $<14,000 \mathrm{~J} / \mathrm{kg}$ per MRI on a first-level controlled MRI mode [13]. Many conditional implants warrant sequence-by-sequence management of energy deposition to reduce heating risks. Mechanisms should be in place to appropriately chart or record SAR/SED values for relevant implants.

\section{Ensure adherence with national guidelines: American College of Radiology}

The ACR Manual on MR Safety specifies MR safety guidelines that provide a basis for the development and implementation of institutional MR policies and practices [14]. As the field of MR safety continues to evolve, these MR safe practice guidelines and subsequent departmental policies and procedures are reviewed and updated regularly [15]. The Medicare Improvements for Patients and Providers Act of
2008 mandates that providers of advanced diagnostic imaging procedures, including MRI, CT and nuclear medicine imaging such as positron emission tomography, be accredited [16]. The United States Centers for Medicare \& Medicaid Services approved three national accreditation organizations: the ACR, the Intersocietal Accreditation Commission and The Joint Commission - to provide accreditation services for suppliers of the technical component of advanced diagnostic imaging procedures. This legislatively mandated accreditation requirement is intended to ensure the reliability, clarity and accuracy of diagnostic images. In addition to fulfilling these requirements, the accreditation process allows facilities to validate the quality of their imaging services [17]. The ACR and the Intersocietal Accreditation Commission are the national regulatory bodies approved by the FDA to accredit MRI performance [17-19]. Therefore, the Safety Council is tasked with incorporating national and accrediting body regulations into the departmental policies and procedures, while preserving image quality and ensuring imaging that surpasses accreditation standards.

\section{Education and communication}

Education of both MR and non-MR personnel is a crucial aspect of any safety program. Training sessions, either online 
or in person, allow for continual reinforcement of safety content and maintenance of qualifications for MR access. According to the ACR safety guidelines [14], MR personnel are typically designated as levels 1 or 2 personnel. Level $1 \mathrm{MR}$ personnel are individuals who have passed the facility's MR safety educational requirements, as determined by the MR medical director. Level $2 \mathrm{MR}$ personnel have received more extensive training and education on the broader aspects of MR safety, including radiofrequency-related burns and neuromuscular excitation. In addition to certification maintenance, regular MR safety training allows for the broad communication and acknowledgment of important updates to policies and procedures.

Communication is the backbone of policies and procedures implementation. In the current health care environment, policy implementation is one of the biggest challenges, as is the accurate, timely and efficient transfer of information among health care workers. Confusion about policy details is one of the most common ways that policies fail, resulting in increased patient risk. Information can be shared in many modalities, and multiple layers of reinforcement are often essential for success. Emails, chat dialogues, posters or intranet postings might be effective for policy changes with a clear, limited plan of action. However, when a more complex policy change requires the execution of multiple synchronous activities, face-to-face in-service meetings provide important opportunities for more advanced training and question-answer sessions. These meetings can also provide important feedback and discussions about potential new issues arising as a result of the implementation. When a new policy or procedural update requires the large-scale re-education of staff both inside and outside the department, a hospital-wide public relations campaign might be important for reinforcing specific behaviors or procedures. Ultimately, personnel training and accurate transmission of information about updates to procedures are crucial to the success of any program.

Safety reporting of MR events or near-misses is a crucial component of any safety program. To ensure accurate information about the strength of the safety program, submissions should be anonymized, and a culture of non-retaliation must be reinforced. Continual education about the importance of safety event reporting is intended to create a culture that views safety reporting as an integral component of patient-centered care, as opposed to punishment or reprisal.

\section{Perspectives from the safety council}

\section{Radiologist perspective}

The specific duties of the members of the MR safety management team are described in detail elsewhere in a consensus document [6]. Briefly, a radiology physician or group of delegated physicians might be designated as MR medical director (MRMD), with the expressed purpose of supervising and coordinating all efforts related to MRI safety. The radiologist's role is to collaborate, as a team member, in the development of MRI-specific safety protocols, policies, quality assurance programs, and assessment of issues that might pose risks to patients and staff.

As a physician, the MR medical director offers important clinical insight concerning the assessment of patient risks and benefits. Expertise in both MRI safety and imaging appropriateness affords the MR medical director a vital perspective toward resolving unique individual safety circumstances (e.g., non-conditional implanted devices). As part of the diagnostic imaging team, a radiologist must be accessible to the MRI technologist performing a study, with the moral and legal duty for the safe performance of an examination residing with both radiologist and technologist. For this reason, all physicians interpreting MRI examinations should be well-versed in the fundamentals of MRI safety to assist their colleagues performing these studies in the event of a safety concern or incident [20]. The MR medical director or delegated radiologist also serves as liaison to referring clinicians and anesthesiologists when safety concerns arise. In the unfortunate event of an MR-related adverse event or near-miss incident, the MR medical director ensures that adequate steps are taken to identify a root cause and develop a strategy to mitigate risk in the future. The ultimate responsibility of physicians involved in MRI safety is to promote a safe MRI environment for patients and staff through reliable communication, collaborative teamwork and efficient coordination of strategies to minimize risk.

\section{Technologist perspective}

The success of MR technologists in upholding their responsibility to ensure a safe MR environment ultimately relies upon the team members. Individuals must have an appropriate understanding of the importance of screening as it pertains to patient, staff and visitor safety. However, they must also be consistent in their safety practices and maintain the ability and willingness to communicate openly and directly with all parties in the MRI suite. Pressure to shortcut the safety process to maintain an efficient workflow is not uncommon; however, it must be met with unwavering commitment to execute all steps as outlined in the safety procedures. Failure to do so, even on a single occasion, presents the potential for a safety breach. Vigilance, regardless of external influence to "keep things moving," is the only means of ensuring safety in the MR environment.

Despite a multitude of physical and cultural barriers, unauthorized entry of ferromagnetic objects into zones 3 and 4 poses a constant and pervasive challenge at many institutions. MR technologists guard zones 3 and 4, permitting entry only to those persons who are appropriately screened. Pediatric MR 
imaging frequently requires multidisciplinary care during any given imaging session. Technologists are responsible for screening every registered nurse, certified registered nurse anesthetist, respiratory therapist, child life specialist and physician who enters zone 3 . Research personnel and the coordination of research studies might also be a source of tension, particularly when scan time on clinical MR scanners is shared with research teams. Because of the risks of unsupervised behavior, all non-MR personnel, including environmental services and security professionals, should not be allowed to be present in the MR scanner environment when the technologists are not present to screen. Safety practices might be challenged, but a confident and factbased explanation by the technologist as the expert in this area should be respected. When this is not the case, an established escalation process should be implemented to ensure support for the technologist from department leadership. As the last line of defense in maintaining MR safety in zone 4, the MR technologist has the ultimate responsibility to ensure that no ferrous items enter the MR scanner room at any time. Their authority as such must be recognized and fully supported. While technologists are the professionals managing and accountable for MRI safety, it is important that institutions attempt to create and support an MR safety culture where all participants share accountability.

In the authors' experience, the biggest threat of safety breaches comes from experienced staff who momentarily lapse in their own consistent and rigorous self-screening. However, near-miss events are important opportunities for strengthening and improving the standard screening processes for all staff, patients and visitors. For example, recognizing the negative impact of pervasive "alarm fatigue" at the threshold of zone 4 , a strict ferrous-free zones 3 and 4 policy can be implemented. One author's institution has also transitioned to require pocketless scrubs for all MR technologists and tech aides, which has mitigated safety risks associated with forgotten items left in pockets. However, ultimately any modifications to the screening process should reaffirm the authority of the MR technologist as the final voice in the decision to provide access to the MR scanner room.

\section{Physicist perspective}

Magnetic resonance imaging has been available in clinical practice for more than 25 years, but in recent times its role in pediatric diagnosis has increased significantly. Furthermore, new techniques and imaging coils for dedicated pediatric use are being developed at a rapid pace. Risks in the MR environment continue to evolve with more frequent use of higher-fieldstrength magnets, higher radiofrequency (RF) frequencies, and more complex equipment. From a practical standpoint, it is difficult to keep up with the implications for interactions between the magnetic field and RF pulses, and the patient and environment. An experienced MR physicist plays a vital role in the continuous evolvement and applications of this diagnostic modality for the care of children. The MR physicist can assist the team in ensuring patient safety by providing safety training and consultation and by actively participating in developing safety policies and procedures [21]. In the role of MR safety expert (MRSE), the MR physicist offers the skill, knowledge and competence to provide high-level advice on the engineering, scientific and administrative aspects of the safe clinical use of MR devices. In particular, the MR safety expert works closely with the MR safety officer and MR medical director to be responsible for (1) the development and continuing evaluation of a safety framework for the MR environment, (2) the development of local rules and procedures to ensure the safe use of MR equipment and (3) the offering of advice regarding non-routine MR procedures for individual patients and specific patient groups.

\section{Conclusion}

A successful pediatric MR safety program serves to comprehensively and iteratively address the safety needs of pediatric patients. Procedure adoption and revision must make sense within the context of the department with respect to workflow and patient care needs. All medical personnel are challenged by the need for vigilance in patient care, despite numerous external pressures. Therefore, any successful program must have the buy-in of all involved parties at each step from scheduling to scanning a patient. They must work as a team, with the unifying goal of caring for children, and must support one another in a constructive manner. Communication is the key to success and is facilitated by creating an open, non-retaliatory environment with respect for individual perspectives.

Despite one's best efforts, no program is immune to safety concerns. However, the success of an MR safety program relies upon relentless team dedication to safety. Policies should be viewed as a live body of knowledge, and the process supporting these policies must adapt to challenges that were not previously defined or understood within an earlier framework. The recent issues related to COVID-19 (coronavirus disease 2019) demonstrate the need for flexibility with changing and evolving demands. However, with appropriate attention to safety concerns, we can proactively address patient care demands and mitigate the risk to our patients.

Acknowledgments We would like to acknowledge the support of several individuals in the creation of this work, including Dr. Elizabeth Drum and nurse Kathryn Crandley for their important insights into these discussions. Additionally, we would like to extend our sincere appreciation for all of the radiologists, technologists, nurses, anesthesiologists, sedation providers, child life specialists, technologist assistants and other MR team members at our institutions. 


\section{Compliance with ethical standards}

Conflicts of interest None

\section{References}

1. Arlachov Y, Ganatra RH (2012) Sedation/anaesthesia in paediatric radiology. Br J Radiol 85:e1018-e1031

2. Serafini G, Zadra N (2008) Anaesthesia for MRI in the paediatric patient. Curr Opin Anaesthesiol 21:499-503

3. Jaimes C, Gee MS (2016) Strategies to minimize sedation in pediatric body magnetic resonance imaging. Pediatr Radiol 46:916-927

4. Siewert B, Swedeen S, Brook OR et al (2018) Barriers to safety event reporting in an academic radiology department: authority gradients and other human factors. Radiology 288:693-698

5. Larson DB, Kruskal JB, Krecke KN, Donnelly LF (2015) Key concepts of patient safety in radiology. Radiographics 35:1677-1693

6. Calamante F, Ittermann B, Kanal E, Norris D (2016) Recommended responsibilities for management of MR safety. J Magn Reson Imaging 44:1067-1069

7. Donnelly LF, Cherian SS, Chua KB et al (2017) The daily readiness huddle: a process to rapidly identify issues and foster improvement through problem-solving accountability. Pediatr Radiol 47:22-30

8. Joshi H, Heilbrun ME, Saindane A et al (2019) Radiology reading room huddles: our initial experience. AJR Am J Roentgenol. https://doi.org/10.2214/ajr.18.20423

9. Choudhri AF, Klimo P Jr, Auschwitz TS et al (2014) 3T intraoperative MRI for management of pediatric CNS neoplasms. AJNR Am J Neuroradiol 35:2382-2387

10. Johnston T, Moser R, Moeller K, Moriarty TM (2009) Intraoperative MRI: safety. Neurosurg Clin N Am 20:147-153

11. McClain CD, Rockoff MA, Soriano SG (2011) Anesthetic concerns for pediatric patients in an intraoperative MRI suite. Curr Opin Anaesthesiol 24:480-486
12. Stienen MN, Fierstra J, Pangalu A et al (2019) The Zurich checklist for safety in the intraoperative magnetic resonance imaging suite: technical note. Oper Neurosurg 16:756-765

13. International Electrotechnical Commission (2002) International standard, medical equipment - part 2: particular requirements for the safety of magnetic resonance equipment for medical diagnosis, 2nd revision. International Electrotechnical Commission, Geneva, pp 2-33

14. American College of Radiology (2020) ACR manual on MR safety, version 1.0. Online document. https:/www.acr.org/-/media/ACR/ Files/Radiology-Safety/MR-Safety/Manual-on-MR-Safety.pdf. Accessed 29 Sep 2020

15. ACR Committee on MR Safety, Greenberg TD, Hoff MN et al (2020) ACR guidance document on MR safe practices: updates and critical information 2019. J Magn Reson Imaging 51:331-338

16. United States Centers for Medicare \& Medicaid Services (2018) Accreditation of advanced diagnostic imaging suppliers. CMS.gov website. https://www.cms.gov/Medicare/ProviderEnrollment-and-Certification/SurveyCertificationGenInfo/ Accreditation-of-Advanced-Diagnostic-Imaging-Suppliers.html.

17. Weinreb J, Wilcox PA, Hayden J et al (2005) ACR MRI accreditation: yesterday, today, and tomorrow. J Am Coll Radiol 2:494-503

18. Serai SD, Jones BV, Podberesky DJ, Coley B (2013) Is it time for a dedicated pediatric MRI ACR accreditation program? J Am Coll Radiol 10:274-278

19. Serai SD, Rigsby CK, Kan HJ et al (2018) Inclusion of pediatricspecific indications and procedures in the new ACR MRI accreditation program. J Am Coll Radiol 15:1022-1026

20. Kanal E, Barkovich AJ, Bell C et al (2013) ACR guidance document on MR safe practices: 2013. J Magn Reson Imaging 37:501-530

21. Mahesh M (2018) Essential role of a medical physicist in the radiology department. Radiographics 38:1665-1671

Publisher's note Springer Nature remains neutral with regard to jurisdictional claims in published maps and institutional affiliations. 